\title{
Breakfast skipping: impact on health parameters and quality of diet in young adults
}

\author{
R.L. Treadwell, I. Alaunyte and F. Amirabdollahian \\ School of Health Sciences, Liverpool Hope University, Liverpool, L16 9JD
}

Previous studies have demonstrated that breakfast is the most important meal of the day ${ }^{(1)}$. Despite this, the evidence suggests that breakfast skipping is a common practice due to the perception that omission of breakfast will reduce energy intake and facilitate weight $\operatorname{loss}^{(2,3)}$. The purpose of this study was to investigate nutritional adequacy and health parameters of healthy young adults by comparing patterns of breakfast consumption $v$ breakfast skipping.

After obtaining ethical approval, mixed gender participants ( $n$ 238, $M$ 104, $F 134)$ aged 18-25 were recruited from universities in the North West of England using convenient and snowball sampling. Anthropometric measurements such as BMI, Waist to Hip Ratio and Body Fat Percentage, and fasting capillary blood lipid profile and glucose were obtained from participant using standardised techniques. Energy and nutrient intake was assessed through the use of a validated 3 day food diary in addition to a short self-reported lifestyle questionnaire which included questions on breakfast skipping patterns. Breakfast skipping was defined as the omission of 2 or more times per week. Normal distribution was analysed using the Shapiro-Wilk test of normality and because the main variables were not normally distributed, measurements were compared using the nonparametric Mann-Whitney $U$ Test. Statistical analyses were conducted using SPSS and statistical significance was set at 0.05.

The results show that half of young adults reported to skip breakfast on regular basis. This trend was seen in both gender groups (males $-50 \%$, females - $51 \%$ ). Body fat percentage median was significantly lower in breakfast consumers than skippers $(23 \% v 26$ $\%, \mathrm{P}<0.05)$. This finding was consistent in males $(18 \% v 15 \%, \mathrm{P}<0.001)$ but not in females $(30 \% v 28, \mathrm{P}=0.067)$. Blood glucose levels were significantly higher in skippers than in consumers. However this trend was not observed in males. Total cholesterol was significantly higher in female breakfast consumers compared to skippers but there were no significant differences in HDL and LDL in both gender groups. Energy and macronutrient intake in breakfast skippers compared to consumers showed no significant trends.

\begin{tabular}{|c|c|c|c|c|c|c|}
\hline \multirow[b]{3}{*}{ Diet and health parameters } & \multicolumn{3}{|c|}{ Breakfast skipper $(n$ 120) } & \multicolumn{3}{|c|}{ Consumer ( $n$ 118) } \\
\hline & & Quartiles & & & Quartiles & \\
\hline & Median & P25 & P75 & Median & $\mathrm{P} 25$ & P75 \\
\hline BMI $\left(\mathrm{kg} / \mathrm{m}^{2}\right)$ & $24 \cdot 0$ & $21 \cdot 6$ & 27.6 & $23 \cdot 4$ & $21 \cdot 2$ & 25.4 \\
\hline$\%$ body fat & $25.9 *$ & $18 \cdot 0$ & 31.6 & $23 \cdot 0^{*}$ & $15 \cdot 4$ & $29 \cdot 3$ \\
\hline Waist to Hip Ratio & 0.80 & 0.74 & 0.85 & 0.80 & 0.74 & 0.85 \\
\hline Total cholesterol $(\mathrm{mmol} / \mathrm{L})$ & $3 \cdot 9^{*}$ & $3 \cdot 3$ & 4.4 & $4 \cdot 2^{*}$ & 3.5 & 4.5 \\
\hline $\mathrm{LDL}(\mathrm{mmol} / \mathrm{L})$ & $2 \cdot 1$ & 1.6 & $2 \cdot 6$ & $2 \cdot 2$ & 1.8 & 2.7 \\
\hline $\mathrm{HDL}(\mathrm{mmol} / \mathrm{L})$ & 1.3 & $1 \cdot 1$ & 1.5 & $1 \cdot 3$ & 1.2 & 1.6 \\
\hline Glucose $(\mathrm{mmol} / \mathrm{L})$ & $4.9 *$ & 4.6 & $5 \cdot 2$ & $4.7 *$ & 4.4 & $5 \cdot 2$ \\
\hline Energy intake $(\mathrm{kJ})$ & $7246 \cdot 5$ & $5820 \cdot 9$ & $9056 \cdot 5$ & 7658.9 & $6107 \cdot 3$ & $9783 \cdot 3$ \\
\hline Carbohydrate (\% TEI) & $45 \cdot 2$ & $39 \cdot 6$ & 49.5 & 44.7 & 39.5 & $49 \cdot 3$ \\
\hline Free sugars (\% TEI) & $15 \cdot 9$ & 11.9 & $20 \cdot 6$ & $16 \cdot 4$ & 11.5 & $20 \cdot 8$ \\
\hline Fat $(\%$ TEI) & 34.9 & $29 \cdot 0$ & 38.4 & $34 \cdot 4$ & 29.5 & 38.3 \\
\hline Saturated fat (\% TEI) & $12 \cdot 2$ & $9 \cdot 1$ & 14.7 & $10 \cdot 9$ & 8.7 & 13.9 \\
\hline Protein (\% TEI) & 17.4 & $15 \cdot 0$ & $20 \cdot 0$ & 18.6 & $15 \cdot 5$ & $23 \cdot 1$ \\
\hline
\end{tabular}

Key: * represent significant $(\mathrm{P}<0 \cdot 05)$ differences between the groups

In conclusion, this study showed that BMI was similar in breakfast skippers and consumers however body fat percentage was significantly higher in breakfast skippers. Blood lipid and glucose profiles showed gender variations. The findings from the present study support previous studies ${ }^{(2,3)}$ suggesting that breakfast skipping as a weight loss tool should not be warranted.

1. Rampersaud, Pereira, Girard et al. (2005) J Am Diet Assoc 105, 743-760.

2. Johns, Tidswell, Mcpherson et al. (2009) J Hum Nutr Diet 22, 256-275.

3. Malinauskas, Raedeke, Aeby et al. (2006) Nutr J 5, 11. 